\title{
COVID-2019: update on epidemiology, disease spread and management
}

\author{
Kamal Kant Sahu', Ajay Kumar Mishra ${ }^{1}$, Amos Lal ${ }^{2}$ \\ ${ }^{1}$ Department of Internal medicine, Saint Vincent Hospital, Worcester, MA; ${ }^{2}$ Division of Pulmonary and Critical care \\ Medicine, Mayo Clinic, Rochester, MN, USA
}

\begin{abstract}
With each passing day, more cases of Coronavirus disease (COVID-2019) are being detected and unfortunately the fear of novel corona virus 2019 (2019-nCoV) becoming a pandemic disease has come true. Constant efforts at individual, national, and international level are being made in order to understand the genomics, hosts, modes of transmission and epidemiological link of nCoV-2019. As of now, whole genome sequence of the newly discovered coronavirus has already been decoded. Genomic characterization nCoV-2019 have shown close homology with batderived severe acute respiratory syndrome (SARS)-like coronaviruses, bat-SL-CoVZC45 and bat-SL-CoVZXC21. Structural analysis of the receptor binding site has confirmed that 2019$\mathrm{nCoV}$ binds with the same ACE 2 receptor protein as human SARS virus. Compared to the previous coronavirus outbreaks, the overall mortality rate is relatively low for COVID-2019 (2-3\%). Suspected cases must be quarantined till their test comes positive or they clear infection. At present, treatment of COVID-2019 is mostly based on the knowledge gained from the SARS and MERS outbreaks. Remdesivir, originally develop as a treatment for Ebola
\end{abstract}

Correspondence: Amos Lal, Division of Pulmonary and Critical Care Medicine, Mayo Clinic, Rochester, MN 55905, USA.

E-mail: manavamos@gmail.com; Amos.Lal@stvincenthospital.com

Conflict of interest: The Authors have no conflict of interest to declare.

Ethical statement: The article does not contain the participation of any human being and animal.

Patient consent: Not applicable.

Contributions: All the authors played a significant role in the paper. All authors have seen the manuscript and agree to the content and data.

Key words: COVID-19; epidemiology; infection; pneumonia; mortality.

Received for publication: 31 Mach 2020.

Accepted for publication: 10 April 2020.

${ }^{\circ}$ Copyright: the Author(s), 2020

Licensee PAGEPress, Italy

Monaldi Archives for Chest Disease 2020; 90:1292

doi: 10.4081/monaldi.2020.1292

This article is distributed under the terms of the Creative Commons Attribution Noncommercial License (by-nc 4.0) which permits any noncommercial use, distribution, and reproduction in any medium, provided the original author(s) and source are credited. virus disease and Marburg virus infections, is being studied for its effectiveness against 2019-nCoV infection. Many other antiviral agents and vaccines are being tested but most of them are in phase I or II and hence unlikely to be of any benefit immediately with regards to current outbreak. Hence, the standard infection control techniques and preventive steps for healthy individuals and supportive care for the confirmed cases is the best available strategy to deal with current viral outbreak.

\section{Introduction}

A novel coronavirus, SARS-CoV-2 has recently been detected (December 2019) amid an outbreak of a cluster of cases of pneumonia of unknown etiology in Wuhan city, Hubei province, China. Within next 8-12 weeks, it has spread globally to the extent that World Health Organization (WHO) declared it as a global pandemic on March 11, 2020. In addition to China, as of $14^{\text {th }}$ March 2020, this pandemic has involved more than 150 countries, with a predominant disease load in Italy, South Korea, Iran, Hong Kong, Macau, and Vietnam. A significant number of deaths have been reported in old individuals and in patients with multiple comorbidities [1]. With so much concern and attention surrounding SARS-CoV-2, knowledge of key clinical information and recent developments is of extreme importance both as a patient and as a health care provider.

\section{Fact and figures related to COVID-19}

From December 2019 till today, many countries worldwide have been affected by the COVID-19 pandemic. As of $14^{\text {th }}$ March 2020, data shows 155,845 confirmed COVID-19 cases with 5,814 deaths worldwide. Currently, there are 75,593 active infected cases, $8 \%(5,908)$ out of that are critically ill. The early few weeks of this outbreak saw China $(80,824$ cases) as having the major burden of the disease. Very soon, countries like, Italy (21,157 cases), Iran $(12,729)$ and South Korea $(8,086$ cases) became the worst-hit countries outside mainland China (Figure 1). Now the number of cases is growing up even due to the $3^{\text {rd }}$ and $4^{\text {th }}$ generation transmissions which signifies that the disease burden is likely going to increase further in coming few weeks.

\section{COVID-19 fatality rate by sex}

Although, we do not have any concrete data, but as per the WHO-China Joint Mission statement dated 28 February 2020, there has been a trend showing more males dying to COVID-19 than females $(4.7 \%$ vs $2.8 \%)$ [2]. However, this data needs to be cautiously interpreted because in China, smoking behavior is more 
commonly seen in the males thereby having more respiratory illnesses and complications.

\section{COVID-19 fatality rate by age}

Older individuals are especially at higher risk of dying from COVID-19 as compared to younger population. The fatality rate significantly worsens in the individuals of more than $60 \mathrm{yrs}(3.6 \%)$ as compared to individuals younger than $40 \mathrm{yrs}(0.2 \%)$.

\section{COVID-19 fatality rate by comorbidities}

Patients who had no pre-existing comorbidities were reported to have a low case fatality rate $(\sim 0.9 \%)$. As, also commonly seen in many other illnesses, pre-existing medical condition increases the risk of dying due to COVID-19 [3-5]. When compared with Wang et al.'s cohort, Huang et al.'s patient population was comparatively younger (median age of 49 versus 56 years) and with a lower fatality rate $(3 \%$ vs $4.3 \%)$. Both studies showed hypertension, diabetes mellitus, and cardiovascular disorders as the most common underlying comorbidities in COVID-19 patients. As per the WHO-China Joint Mission statement (dated 28 February 2020) [2], a risk of dying of a confirmed COVID-19 patient is highest by having cardiovascular disease (13.2\%), diabetes mellitus $(9.2 \%)$, hypertension $(8.4 \%)$, and chronic respiratory disease $(8 \%)$ in decreasing order.

\section{Basic (R0) and effective reproduction number (R) of SAR-CoV-2}

The basic reproduction number (R0) is a useful predictor that measures the transmission capability of a disease. It is defined as an average number of secondary infections produced by one case in a population considering everyone is susceptible. However, in a practical world, it is rare to have a population to be completely susceptible to a given infection due to the presence of immune individuals as well. In order to successfully wipe out a disease, $\mathrm{R}$ should go down below 1. With regards to SARS-CoV-2, there has been a substantial variation in reporting of transmission rates over time [6,7]. Liu et al. recently reviewed 12 studies on R0 for COVID-19 and found that the major reason for the difference in reported $\mathrm{R} 0$ is probably due to the various estimation methods used While analyzing the results [8]. They found that the studies using mathematical methods estimated a higher average R0 of 4.2 (range: 1.5 to 6.49) [9-13]. A reasonably comparable R0 was found with studies using stochastic (average R02.44, range: 2.2-2.68) and statistical methods (average R0- 2.67, range: 2.2 to 3.58$)[6,14-16]$.

\section{Virology and epidemiology of SARS-CoV-2}

\section{Taxonomy}

In general, coronaviruses are abundant in birds and mammals and constitute a big family of non-segmented, enveloped, positivesense, single-stranded RNA viruses [17]. Amongst these, bats are believed to be the home to the greatest variety of genotypes. Combined, animal and human coronaviruses fall into four genera namely alpha coronavirus, beta coronavirus, gamma coronavirus, and delta coronavirus genus. The Middle East respiratory syndrome (MERS), Severe acute respiratory syndrome (SARS), and SARS-CoV-2 all three belong to beta coronavirus genus.

\section{Virus receptor-binding domain-Human ACE-2 receptor interaction}

Atomic-level of understanding of SARS-CoV-2 and its interaction with human cells has considerably clarified the pathogenesis of COVID-19 disease at a molecule are level. Scientists have succeeded in recognizing the receptor i.e. ACE-2 receptor for the attachment of SARS-CoV-2. Wan et al. from the college of Veterinary Medicine, Minnesota studied the complex interaction of

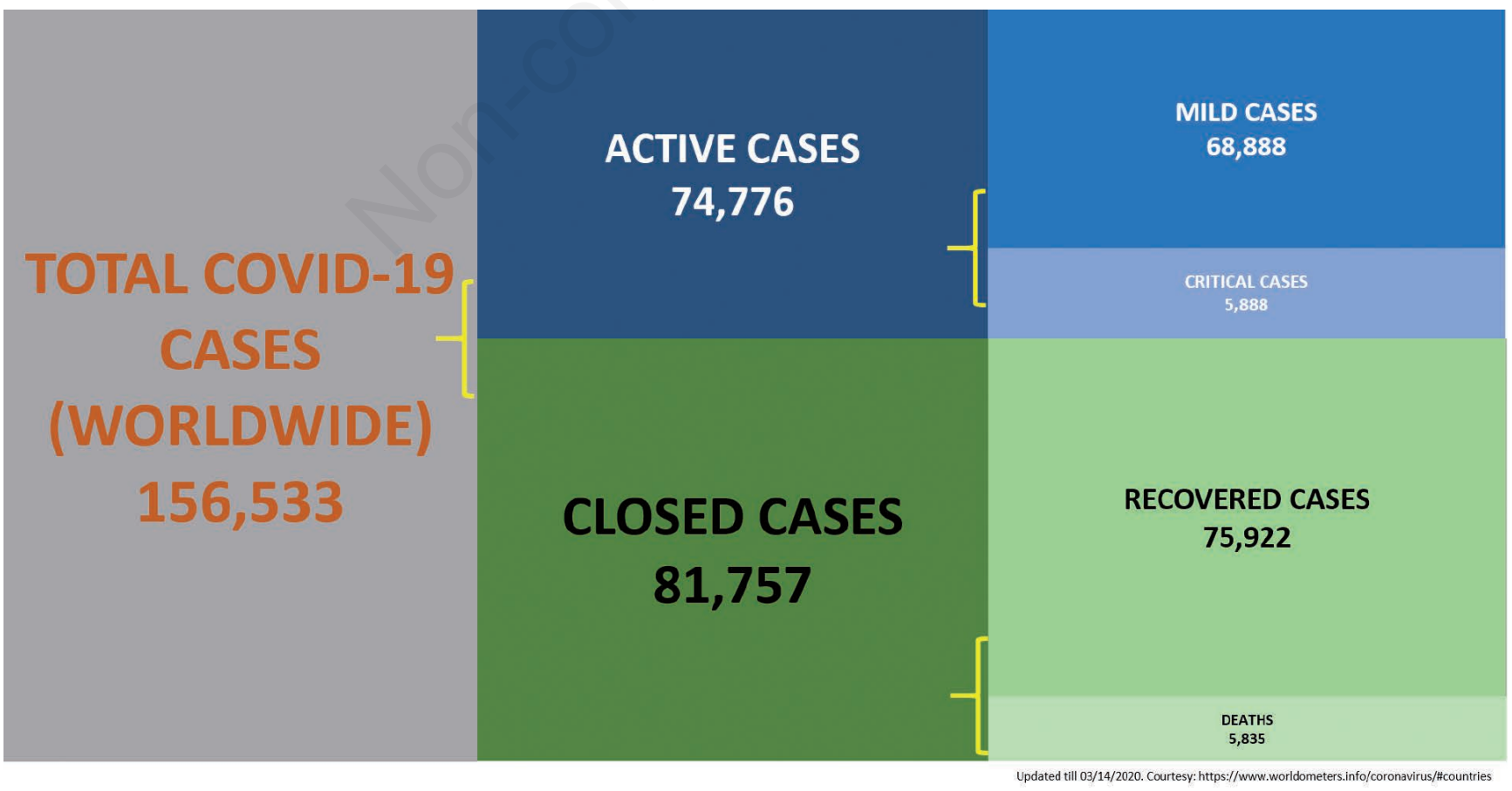

Figure 1. Total number of cases worldwide with detailed breakdown of active and recovered cases. 
SARS-CoV-2 receptor-binding domain (RBD) with the ACE-2 receptor. Based on their structure-function protective framework, they concluded that ACE-2 receptor for SARS-CoV-2 is the same as for the SARS virus responsible 2003-2004 outbreak [18]. They studied various virus binding hot spots on human ACE 2 receptors and compared with orthologues isolated from other species. They found that amino acids located at 442, 472, 479, 480 and 487 positions enhance the viral binding capacity to the human ACE-2 receptors. Similarly, a single mutation N501T was found to enhance the interaction between SARS-CoV-2-RBD with the ACE-2 receptor. Data suggests that SARS-CoV-2 binds to human ACE 2 receptors even more efficiently than the SARS-CoV-2003 strain but less efficiently than the SARS-CoV-2002 strain. Similar studies are underway with the long-term goal of understanding epidemiological link, identifying the potential definite and intermediate hosts, and predicting the species-specific interactions and susceptibility to these viral infections.

\section{Incubation period}

According to the Centers for Disease Control and Prevention (CDC), the mean incubation period of COVID-2019 is approximately 5.1 days (range 2-14 days). Lauer et al. did a pooled analysis of 181 confirmed COVID-19 cases from China [19]. They estimated the median incubation period of COVID-19 to be 5.1 days. The study showed that $95 \%$ of patients had incubation period ranging from 2.1 (CI, 1.5 to 3.2 ) to 14.7 (CI, 7.4 to 22.6 ) days. These numbers justify the quarantine period of 14 days set by the World Health Organization (WHO). It is important to understand that for any communicable disease, latent period and incubation period are two different entities affecting the transmission dynamics. Based on the discussion by Lauer et al., it is very much possible that the latency period of COVID-19 may be less than its incubation period there by meaning, people may be contagious even before being symptomatic [19].

\section{Mode of transmission}

It is easy to understand the concept of being symptomatic and contagious to transmit a pathogen to healthy individuals in case of a communicable disease [20]. However, now there are recent evidences of SARS-CoV-2 transmission by even minimally symptomatic or asymptomatic individuals $[21,22]$. As of now, the routes of SARS-CoV-2 transmission seems to be diversified. Major transmission routes are through close or direct contact with infected secretions or large aerosol droplets [23]. There is a growing concern over the possibility of the role of fecal-oral transmission in COVID $\square 19$ transmission [24,25]. The obvious logic behind this speculation is the fact that ACE-2 receptor protein is also found in abundance in the epithelia of intestinal lumen. Also, Zhang et al. from Wuhan University detected the SARS-CoV-2 viral nucleic acids in the fecal samples and anal swabs of COVID $\square 19$ patients [24]. The brighter side is that as compared to MERS and SARS, patients with COVID19 have reported lesser gastrointestinal symptoms-diarrhea ( $\square \square 10.1 \%$ ), and nausea and vomiting ( $1 \square 3.6 \%)$. Fecal-oral route is known to be responsible for many endemic diseases especially in developing countries [26]. The exact significance of gut-lung crosstalk and the role of gut microbiota in COVID-19 is yet to be determined. Hence, more definitive evidence is required before we can say that targeting gut microbiota would fetch as a new therapeutic option. With regards to vertical transmission in pregnant woman from mother to baby, none of the studies conducted so far on COVID-19 affected pregnancies have shown any evidence of vertical transmission, viral shedding in the vaginal secretions, or evidence of SARS-CoV-2 in breast milk [27-29].

\section{Definitive and intermediate hosts}

Like the previous coronavirus outbreaks, there is enough evidence now that the virus responsible for the COVID-19 pandemic has a hidden reservoir in the wild animals and possibly there were accidental spillover to human population leading to this outbreak (Figure 2) [30,31]. Phylogenetic analysis and virus genome analysis with annotation have shown that coding regions of SARS-CoV-2 possess a similar genomic structure to bat-SLCoVZC45, bat-SL-CoVZXC21, and SARS-CoV [32]. Paraskevis et al. also did a complete genome evolutionary analysis of the SARS-CoV-2 and confirmed similar findings with $96.3 \%$ sequence similarity to the BatCoV RaTG13 sequence. Interestingly, they also found evidence of discordant clustering in SARS-CoV-2 with the Bat SARS-like coronavirus sequences. They concluded saying SARS-CoV-2 likely originated from bats but rejected the possibility of this outbreak as a result of any recent recombination event [33]. Hence, while the exotic animals are the definitive hosts, humans and domestic animals get accidentally exposed to wild animals during their intentional or unintentional exposure and interaction with them.

\section{Clinical presentation}

\section{Asymptomatic infections}

During the initial days of outbreak, only the symptomatic individuals, were thought to be infectious and capable of transmitting the infection. However very soon, convincing studies came up which suggested that asymptomatic individuals could potentially also be transmitting the infection. Hu et al. studied the clinical

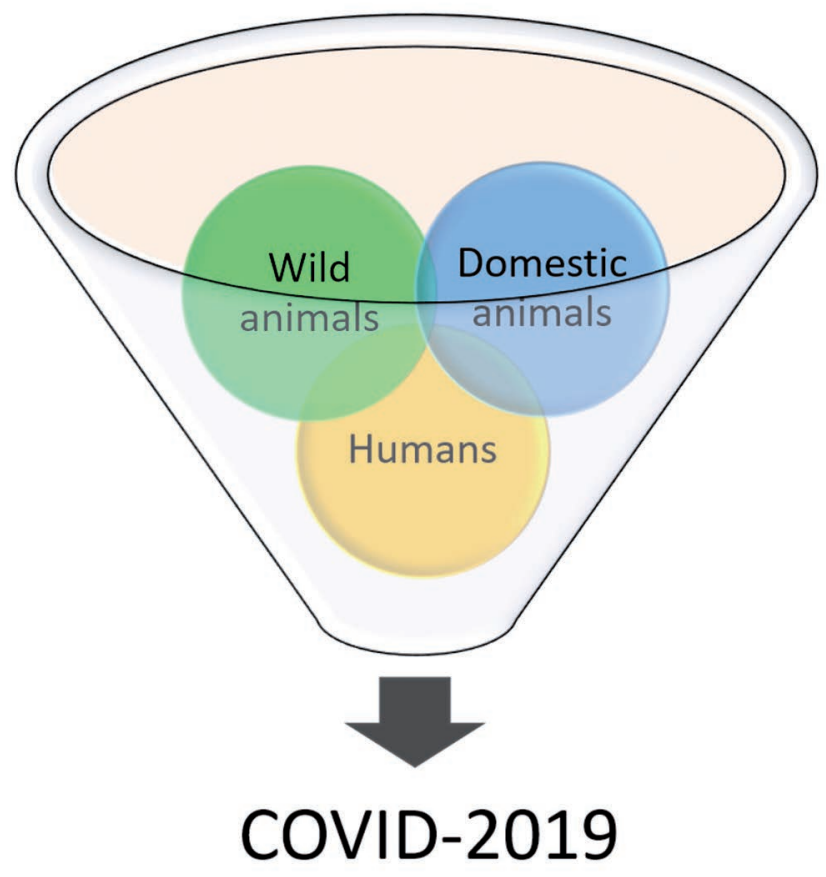

Figure 2. Epidemiological link and cross talk between wild animals, domestic animals and humans leading to spillover of the coronavirus to humans. 
characteristics of 24 asymptomatic individuals who were found positive for SARS-CoV-2 while screening for COVID-19 [34]. At follow up, only five individuals $(20.8 \%)$ developed symptoms (fever, cough, fatigue, etc.). $70.8 \%$ of individuals had CT imaging shadows (twelve reported typical GGOs, five reported as stripe shadowing in the lungs). These results underscored the importance of close contact tracking and lengthwise reconnaissance via appropriate virus nucleic acid tests.

\section{Symptomatic patients}

Data on clinical presentation of the SARS-CoV-2 outbreak is rapidly evolving. The commonly reported symptoms as per the earlier studies were predominantly related to the respiratory system like fever, dry cough, fatigue, and myalgia $[4,5]$. Non-respiratory symptoms like nausea, vomiting, and diarrhea were uncommon presentations. Unfortunately, there are no pathognomonic clinical features that could confidently differentiate COVID-19 from other routine viral respiratory infections $[35,36]$. The problem seems to be worsening as we are moving away from $1^{\text {st }} / 2^{\text {nd }}$ generation transmissions to $3^{\text {rd }}$ and $4^{\text {th }}$ generation transmissions. As per the study by Chen et al on 89 COVID-19 patients from Wuhan, the patients presenting before $23^{\text {rd }}$ January 2020 had more systemic symptoms and productive cough as compared to the patients admitted after $23^{\text {rd }}$ January 2020 [37]. This indicates that, the pattern of symptomatology is also evolving gradually with time and hence we should be watchful for this change in symptomatology pattern so that the screening criteria can be changed if required.

\section{Investigations}

A diagnostic test capable of rapid and accurate detection of SARS-CoV-2 is the need of the hour due to the enormous spread of pandemic and increasing number of COVID-19 patients. With regards to the United States, any suspected patient should be reported immediately to appointed infection-control authority at the local health center and then to the local or state health department. Currently, in the United States, CDC is the only center where the diagnostic testing can be conducted officially. Once the State health department receives a call from any Medical Center, it is required to immediately contact CDC's Emergency Operations Center (EOC) at 770-488-7100 who will then assist with the collection, storage, and shipment of the clinical specimens.

\section{Reverse transcription polymerase chain reaction (RT-PCR)}

As per CDC guidelines, a reverse-transcription polymerase chain reaction (RT-PCR) test is the current standard of test to confirm COVID-2019. However, RT-PCR has also its own challenges like i) delay in result turnaround time, and ii) interpreting the results. Another major hurdle is the dynamic conversion of RTPCR results from either negative to positive or from positive to negative. Hence, combining RT-PCR with other investigation like computed tomography (CT) of the chest in an appropriate clinical setting is the best modality to investigate any patient. Ai et al. in their recent study suggested a higher sensitivity of CT chest $(98 \%)$ than RT-PCR (71\%) in diagnosing COVID-2019 [38].

\section{Choosing the right specimen}

COVID-19 disease is new for everyone and hence with each passing day, more information is pouring in with regards to the diagnostic approach and selecting the best type of specimen to run the test. Elucidating the diagnostic accuracy of various respiratory specimens holds a pivotal role in the control, diagnosis, and treat- ment of COVID-19 disease. Specimens can be collected from the upper respiratory tract (nasopharyngeal and oropharyngeal swabs), lower respiratory tract (sputum, endotracheal aspirate, bronchoalveolar lavage), serum and urine and stool if possible.

Collection samples from the lower respiratory tract (usually BAL) are not feasible always in every case due to logistic and medical reasons. Hence, BAL samples are not a routine laboratory sample available for diagnosis and monitoring of the SARS$\mathrm{CoV}-2$. Instead, the collection of samples from the upper respiratory tract like a nasal swab, throat swab and sputum are rapid, simple and safe. Yang et al studied a total of 866 samples from 61 respiratory tracts of the patients including nasal swabs, throat swabs, sputum, and 62 BAL fluid samples. Amongst the upper respiratory tract samples, their study showed a sputum sample having the highest positive rate (74.4-88.9\%), followed by nasal swabs (53.6-73.3\%) for both severe and mild cases. They recommended whenever available, monitoring viral RNA titers in the BAL fluids could help in understanding the disease trajectory and prognosis $[39,40]$.

Also, it is important to remember that the CDC does not recommend any virus isolation in cell culture due to biosafety reasons. Few studies have suggested that a COVID-2019 is ruled out in any case if RT-PCR is negative from respiratory tract samples on two consecutive occasions which are 24 hours apart. But as discussed above, RT-PCR alone can have a low detection rate [38,41]. Also, to note that all respiratory samples should also be tested for the usual viruses such as influenza type A, influenza type B, adenovirus, respiratory syncytial virus and so on.

\section{Computed tomography (CT) of chest}

With many limitations of RT PCR, the role of imaging is increasingly recognized as a crucial step in diagnosing suspected cases of COVID-19. When compared to RT-PCR, CT imaging seems to be feasible, easily available and a rapid method. A higher sensitivity rate of CT scan (97\%) than RT PCR (71\%) puts CT in a driving position with regards to COVID-19 diagnosis [38,41]. As reported by most studies, the common CT imaging findings are ground-glass opacities (GGOs), consolidation, bilateral/multilobar involvement, peripheral distribution, round opacities and interlobular thickening $[4,5,42]$. Linear opacities, crazy paving, and reverse halo have been also reported as common late findings as disease progresses. In contrast, findings which are considered inconsistent or atypical for COVID-19 infection are tree-in-bud opacities, centrilobular distribution, peribronchovascular distribution, cavitation, lymphadenopathy, pleural effusion and predominant nodular opacities [41].

\section{Laboratory testing}

With regards to laboratory workup, the common laboratory studies are complete blood count, coagulation profile, serum biochemical tests (including renal and liver function, creatine kinase, lactate dehydrogenase, and electrolytes), myocardial enzymes, and procalcitonin. The most common reported laboratory findings so far reported are normal/ low white cell counts, elevated C-reactive protein (CRP), deranged coagulogram, and elevated lactic acid dehydrogenase. Zhou et al. reviewed 191 COVID-19 patients and found that as compared to survivors, the non-survivors had lower baseline lymphocyte count, which continued to worsen until the day of death [43]. Similarly, non-survivors had higher d-dimer, high-sensitivity cardiac troponin I, serum ferritin, lactate dehydrogenase, and IL-6 values in comparison to the survivors. Similar results were also reported by Fan et al. who reviewed 69 patients and found that, ICU 
requiring patients ( $\mathrm{n}=9$ cases) were comparatively a decade older, and had significant lymphopenia $(\mathrm{p}<0.001)$, significant neutrophilia $(p<0.001)$, significant elevated LDH $(p<0.005)$ and significantly lower CD45+, CD3+, CD4+, CD19+, and CD16/56+ counts when compared with non-ICU patients ( $\mathrm{n}=58$ cases) [44].

\section{Prevention}

To date, no effective vaccination is available. The risk of acquiring secondary and atypical infections is more so in cases of immunocompromised individuals [45]. Therefore, the best way in order to protect oneself is to follow the conventional infection control protocols and avoid unnecessary travel, public transport, contact with sick people and so on.

\section{Significance of hand washing}

The importance of frequent and proper hand hygiene should be emphasized. SARS-CoV-2 like the other coronaviruses have a lipid envelope and in simple words, washing with soap can break that fat in the envelope apart and thereby making it impossible or difficult for the virus to infect human cells. Hence, handwashing with soap and water is by far the more powerful weapon than any other preventive measure. Also, more importantly, the duration of the soap wash is also equally important. CDC recommends at least 20 seconds of effective engagement in hand washing. In a recent study on field observations of 3,749 people in a college town environment, Borchgrevink et al. found that only about $5 \%$ of them followed all the rules of handwashing and only 5\% engaged themselves in an effective handwashing technique (washing, rubbing, and rinsing) for more than 15 seconds [46]. This indicates that, understanding of the public regarding the importance of hand washing in general is poor and hence every attempt should be made to reinforce to adopt this essential preventive measure in day-to-day practice.

\section{Appropriate use of face mask}

Physical barrier in the form of using a face mask by individuals showing respiratory symptoms is recommended by WHO. Healthy individuals are not advised to use a face mask. A surgical mask provides only "one-way protection" and prevents the spreading of droplets during sneezing and coughing from a wearer to the surrounding areas (Figure 3). In contrast, health care providers who are taking care of suspected or proven cases of COVID-19 must wear a specialized respirator, N95 which technically is a good fit mask preventing the entry of droplets and thereby minimizing the chance of acquiring the infection. Considering the high risk of transmission through direct contact, stringent precautions should be displayed especially while handling the body secretions like urine, sputum or stools of the affected patients, and sewage from hospitals [47]. WHO on $3^{\text {rd }}$ March 2020, released a statement requesting various industries and governments to increase the production of personal protective equipment (PPEs) by at least $40 \%$ to meet rising global demand. The shortage of PPEs is an immediate threat to the frontline health care providers endangering their life.

\section{Treatment}

\section{Current standard of care}

At present, there is no specific antiviral agent that is approved for COVID-19. Hence, supportive care to help alleviate symptoms is the best current approach being followed by all the medical centers worldwide. Supportive care includes isolating the patient to a negative pressure isolation room, and providing adequate rest, hydration, nutritional support and electrolyte balance. Complicated cases developing respiratory failure, ARDS, heart failure and septic shock also require a high level of care and other life support like invasive ventilation, extracorporeal membrane oxygenation (ECMO), renal replacement therapy and so on.

\section{Facts about Indications of Wearing a Mask}

\section{Surgical Mask}

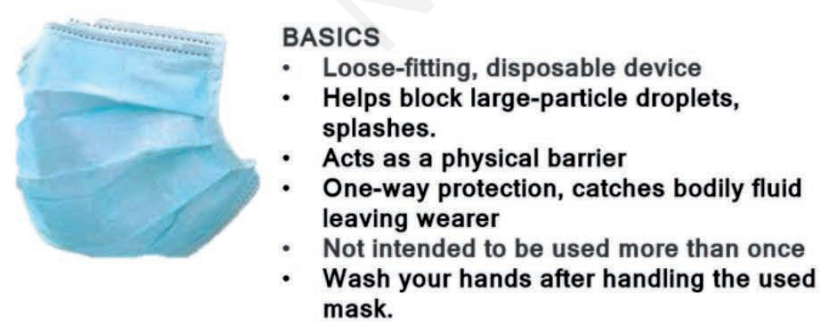

N 95 Respirator

BASICS

- Very close facial fit

- Efficient filtration of airborne particles.

- 'N95' means $95 \%$ of very small ( 0.3 micron) particles

- Two-way protection, it filters the air breathed in.

- One-time use, disposable

- Not designed for children or with facial hair
Who should wear a Surgical mask?

If a person has respiratory symptoms, like cough, sneeze or shortness of breath.

$\checkmark$ Not needed for general population who are healthy and without any respiratory symptoms.

\section{Who should wear a N 95 Respirator?}

$\checkmark \quad$ If a person is a health care provider and providing care to a patient with has respiratory symptoms.

$\checkmark$ Not needed for general population who are healthy or even with respiratory symptoms.

Figure 3. Description of surgical mask and N 95 Respirator with indications. 


\section{Current stand of antiviral agents}

As mentioned above, as of now there is no specific antiviral therapy for COVID-19 [48-55]. However, there are antiviral agents that were found effective during the previous outbreaks of SARS and MERS. Amongst the commonly used antiviral agents, ribavirin, interferon, lopinavir-ritonavir, have been used in the past in patients with SARS or MERS. These antiviral agents are currently being used either alone or in various combinations by physicians in various medical centers worldwide to treat their COVID-19 cases, although the exact efficacy is still unclear. Hence, substantial efforts are ongoing to find out new therapeutic agents for this coronavirus infection. Hence whenever possible, patients should be enrolled in ongoing randomized control trials. We encourage physicians to review the latest developments and available evidence while considering using any agent as anti-SARS-CoV-2 medication. It is also not clear till now whether a single drug versus a combination of multiple anti-viral agents would be appropriate to treat COVID-19 cases.

\section{Current stand of antibacterial agents}

Antibacterial agents are not to be used empirically. Only in cases where a secondary bacterial infection/pneumonia is suspected, an antibacterial agent like cephalosporins and fluoroquinolones should be used after sending blood cultures [56].

\section{Role of corticosteroids}

Ideally speaking, the current stand of corticosteroid use in COVID-19 is controversial due to the concern that it may delay the viral clearance from the body. However, practically speaking a judicial short term use of steroids (methylprednisolone 1-2 mg/kg body weight per day for 3-5 days) is being used frequently by various health care centers worldwide for severe cases in order to ameliorate lung inflammation to prevent the development of acute respiratory distress syndrome.

\section{Use of Traditional Chinese medications}

Traditional Chinese Medicine (TCM) has been used since ancient times to treat a variety of disorders across the globe [57,58]. China's National Health Commission and National Administration of Traditional Chinese Medicine recently recommended using probiotics in the treatment of severe COVID $\square 19$ [57]. This could possibly maintain a balance of intestinal microecology and prevent secondary bacterial infection. However, it should only be used as an ancillary add on therapy in addition to the mainline treatment strategy.

\section{Newer drugs, vaccines and clinical trials}

A coalition of researchers and scientists worldwide are working relentlessly with an aim to develop an ideal antiviral agent and vaccine against SARS-CoV-2. In general, antiviral agents could belong to be a i) Virus-based therapy like monoclonal antibodies or antiviral peptides targeting the various molecules, steps or levels of viral machinery like viral spike glycoprotein, viral enzyme inhibitors, and viral nucleic acid synthesis inhibitors, or ii) Hostbased therapies to potentiate the interferon response, affect host signaling pathways, or host factors utilized by SARS-CoV-2 for replication [52].

There are scientists who are also working on the concept of drug repurposing, which is a strategy to generate an additional ben- efit from an existing drug to target other new diseases [48,49]. For instance, the concomitant actions of ezetimibe and statins might be useful for the treatment of several viral infections (NCT00908011, NCT00099684, NCT00843661, NCT03490097, NCT00994773, NCT00441493). On similar lines of drug repurposing, Wang et al recently studied ribavirin, penciclovir, nitazoxanide, nafamostat, chloroquine and two broad-spectrum antiviral drugs Remdesivir (GS-5734) and favipiravir (T-705) for their efficacy against SARS$\mathrm{CoV}-2$ in vitro. They found that Remdesivir, nitazoxanide, and chloroquine were able to block SARS-CoV-2 infection at a lowmicromolar concentration [51]. Remdesivir is a nucleotide analog that was originally developed during the Ebola outbreak and has shown superior antiviral activity to lopinavir (LPV) and Ritonavir (RTV) [54]. Unfortunately, it is not commercially available and has been used only on compassionate ground in a patient with COVID19 in the United States [53]. In contrast, LPV/RTV combination is widely available with a predictable toxicity profile but in a recent 18 patient series from Singapore, 5 cases who were treated with LPV/RTV combination had variable treatment outcomes and significant toxicities of nausea, vomiting, diarrhea, and hepatotoxicity [55]. National Institutes of Health (NIH) has initiated a clinical trial to test Remdesivir for its clinical effectiveness at the University of Nebraska Medical Center (UNMC) in Omaha (NCT04280705) [57].

\section{Special scenarios: Need of tailored approach}

\section{Pregnancy and special needs}

Having a disease while being pregnant is a special situation as now the body has to deal with a physiological stress related to pregnancy and an additional perturbation due to pathological disease [27,59]. The treatment of COVID-19 pregnant patients is more challenging than non-pregnant cases with certain additional precautions. The management requires a multi-disciplinary approach including obstetric-gynecologists, neonatologists, intensivists, infectious disease specialists, and internists. If required, a CT scan of the chest should be performed as the fetal risk of radiation exposure is considered very small. Secondly, amongst the antiviral agents, LPV/RTV combination is preferable to use considering their safety profile in pregnancy [60-62]. Ultrasound examination for monitoring fetal well-being is also recommended. If a preterm delivery is anticipated, an intramuscular betamethasone injection should be given to ensure fetal lung maturity. There is no evidence of viral shedding through vaginal secretions or vertical transmission of SARS-CoV-2. Hence all patients if clinically stable should undergo vaginal delivery $[27,63]$. There are recent reports of post-natal transmission of the infection from diseased mother to the newborn. Considering this, an early cord clamping is recommended. It is recommended to separate the newborn from COVID-2019 infected mother for at least two weeks. Also, during this period of separation, breastfeeding is not recommended [27].

\section{Cancer patients and their care}

Patients suffering from cancer are at higher risk of acquiring viral infections like SARS-CoV-2 infection and are likely to develop more complications $[64,65]$. Patients with hematological malignancies, patients on active chemotherapy, neutropenic patients, and bone marrow transplant patients are significantly at a higher risk [66]. Almost all major cancer centers and oncology experts have 
issued their respective guidelines and statements for their practicing oncologists, nurse practitioners, cancer patients, and their care providers.

In addition to following the general guidelines of personal and social etiquettes, cancer patients should take a special precaution like i) get a few extra refills of the essential medications; ii) discuss with primary oncologist before making any travel plans; iii) reschedule all the non-urgent appointments for the later date; iv) discuss with the primary oncologist about the feasibility of rescheduling/postponing any maintenance chemotherapy/immunotherapy in an otherwise well-controlled cancer in remission; v) ensure that oneself is up to date on a vaccination schedule.

\section{Cardiovascular patients with COVID-19 disease}

Most studies have showed patients with cardiovascular risk factors like advanced age, hypertension, diabetes, cardiovascular diseases, cerebrovascular disease had washed clinical outcome [3]. A recent study including 22,000 patients from Italy, reported mortality of $7.2 \%$. Cardiovascular risk factors in term of diabetes, ischemic heart disease, atrial fibrillation and a history of stroke was present in $35.5 \%, 30 \%, 24.5 \%$, and $9.6 \%$ simultaneously among a subset of patient with mortality. Advanced age was associated with higher mortality in this study with a reported case fatality of $12.8 \%, 20.2 \%$ in patients above age group of 70 and 80 simultaneously. Across all studies cardiovascular risk factors like advanced age, hypertension, diabetes, coronary artery disease, heart failure, atrial fibrillation, cerebrovascular disease have been reported to be associated with increased mortality [67,68]. As shown in image one these factors have been independently shown to be associated with worsening disease severity, bilateral confluent lung involvement on CT chest involvement of multiple organs, requirement of invasive and noninvasive ventilation, presence of cardiac injury, and mortality [69-71].

\section{Conclusions}

Our current understanding of COVID-19 is limited and is still evolving. COVID-19 disease burden has put a lot of pressure on health resources and patient care. Elimination of COVID-19 is an international priority because, without control of viral spread, the disease burden is going to increase every day with increased mortality. Till now, the best proven measures are infection control policies of rigorous contact tracing, stringent quarantine of symptomatic contacts, and hospital isolation and screening of symptomatic cases.

\section{Bibliography}

1. Sahu KK, Mishra AK, Lal A. Comprehensive update on current outbreak of novel coronavirus infection (2019-nCoV). Ann Transl Med 2020. doi: 10.21037/atm.2020.02.92.

2. WHO. Report of the WHO-China Joint Mission on Coronavirus Disease 2019 (COVID-19). World Health Organization. Available from: https://www.who.int/docs/ default-source/coronaviruse/who-china-joint-mission-oncovid-19-final-report.pdf

3. Sahu KK, Mishra AK, Lal A, Abraham GM. Mycobacterium avium complex: A rare cause of pancytopenia in HIV infection. J Microsc Ultrastruct 2019;8:27-30.
4. Wang D, Hu B, Hu C, et al. Clinical characteristics of 138 Hospitalized patients with 2019 novel coronavirus-infected pneumonia in Wuhan, China. JAMA 2020;e201585. doi: 10.1001/jama.2020.1585.

5. Huang C, Wang Y, Li X, et al. Clinical features of patients infected with 2019 novel coronavirus in Wuhan, China. Lancet 2020;395:497-506. doi: 10.1016/S0140-6736(20)30183-5

6. Li Q, Guan X, Wu P, et al. Early transmission dynamics in Wuhan, China, of novel coronavirus-infected pneumonia. N Engl J Med 2020 Jan 29.

7. Kucharski AJ, Russell TW, Diamond C, et al. Early dynamics of transmission and control of COVID-19: a mathematical modelling study. Lancet Infect Dis 2020. doi: 10.1016/S14733099(20)30144-4.

8. Liu Y, Gayle AA, Wilder-Smith A, Rocklöv J. The reproductive number of COVID-19 is higher compared to SARS coronavirus. J Travel Med 2020;27(2):taaa021. doi: 10.1093/jtm/ taaa021.

9. Shen M, Peng Z, Xiao Y, Zhang L. Modelling the epidemic trend of the 2019 novel coronavirus outbreak in China. bioRxiv 2020. doi: https://doi.org/10.1101/2020.01.23.916726.

10. Read JM, Bridgen JRE, Cummings DAT, et al. Novel coronavirus 2019-nCoV: early estimation of epidemiological parameters and epidemic predictions. medRxiv 2020. doi: https://doi.org/10.1101/2020.01.23.20018549.

11. Majumder M, Mandl KD. Early transmissibility assessment of a novel coronavirus in Wuhan, China. Accessed on: 27 January 2020. Available from: https://papers.ssrn.com/abstract=3524675

12. Cao Z, Zhang Q, Lu X, et al. Estimating the effective reproduction number of the 2019-nCoV in China. medRxiv 2020. doi: https://doi.org/10.1101/2020.01.27.20018952.

13. Imai N, Cori A, Dorigatti I et al. Report 3: transmissibility of 2019-nCoV. 2020. WHO Collaborating Centre for Infectious Disease Modelling, MRC Centre for Global Infectious Disease Analysis, J-IDEA, Imperial College London, UK.

14. Wu JT, Leung K, Leung GM. Nowcasting and forecasting the potential domestic and international spread of the 2019-nCoV outbreak originating in Wuhan, China: a modelling study. Lancet 2020;395:689-97. doi: 10.1016/S0140-6736(20)30260-9.

15. Zhao S, Ran J, Musa SS, et al. Preliminary estimation of the basic reproduction number of novel coronavirus (2019-nCoV) in China, from 2019 to 2020: a data driven analysis in the early phase of the outbreak. bioRxiv 2020. doi: https://doi.org/ 10.1101/2020.01.23.916395.

16. Riou J, Althaus CL. Pattern of early human-to-human transmission of Wuhan 2019-nCoV. bioRxiv 2020. Accessed on: 27 January 2020. Available from: https://www.biorxiv.org/content/10.1101/2020.01.23.917351v1.full.pdf

17. Sahu KK, Mishra AK, Lal A. Latest updates on COVID-2019: A changing paradigm shift. J Med Virol 2020. doi: 10.1002/jmv.25760. [Epub ahead of print].

18. Wan Y, Shang J, Graham R, Baric RS, Li F. Receptor recognition by novel coronavirus from Wuhan: An analysis based on decade-long structural studies of SARS. J Virol 2020. doi: 10.1128/JVI.00127-20.

19. Lauer SA, Grantz KH, Bi Q, et al. The incubation period of coronavirus disease 2019 (COVID-19) from publicly reported confirmed cases: Estimation and application. Ann Intern Med 2020;M20-0504. doi: 10.7326/M20-0504.

20. Lal A, Al Hammadi A, Rapose A. Latent tuberculosis infection: Treatment initiation and completion rates in persons seeking immigration and health care workers. Am J Med 2019;132:1353-5. 
21. Chan JF, Yuan S, Kok KH, et al. A familial cluster of pneumonia associated with the 2019 novel coronavirus indicating person-to-person transmission: a study of a family cluster. Lancet 2020;395:514-23.

22. Rothe C, Schunk M, Sothmann P, et al. Transmission of 2019nCoV infection from an asymptomatic contact in Germany. N Engl J Med 2020;382:970-1. doi:10.1056/NEJMc2001468.

23. Yang HY, Duan GC. [Analysis on the epidemic factors for the Corona Virus Disease].[Article in Chinese]. Zhonghua Yu Fang Yi Xue Za Zhi. 2020;54:E021.

24. Zhang W, Du RH, Li B, et al. Molecular and serological investigation of 2019-nCoV infected patients: implication of multiple shedding routes. Emerg Microbes Infect 2020;9:386-9.

25. Gao QY, Chen YX, Fang JY. 2019 novel coronavirus infection and gastrointestinal tract. J Dig Dis 2020;21:125-6.

26. Lal A, Davaro R, Mishra AK, et al. Detection of coexisting toxigenic Clostridium difficile and nontyphoidal Salmonella in healthcare worker with diarrhea: A therapeutic dilemma. J Family Med Prim Care 2019;8:2724-7.

27. Liang H, Acharya G. Novel corona virus disease (COVID-19) in pregnancy: What clinical recommendations to follow? Acta Obstet Gynecol Scand 2020;99:439-42. doi: 10.1111/ aogs. 13836.

28. Liu Y, Chen H, Tang K, Guo Y. Clinical manifestations and outcome of SARS-CoV-2 infection during pregnancy. J Infect 2020. pii: S0163-4453(20)30109-2.

29. Favre G, Pomar L, Musso D, Baud D. 2019-nCoV epidemic: what about pregnancies? Lancet 2020;395:e40.

30. Kemenesi G, Dallos B, Görföl T, et al. Molecular survey of RNA viruses in Hungarian bats: discovering novel astroviruses, coronaviruses, and caliciviruses. Vector Borne Zoonotic Dis 2014;14:846-55.

31. Sharmin R, Islam AB. Conserved antigenic sites between MERS-CoV and Bat-coronavirus are revealed through sequence analysis. Source Code Biol Med 2016;11:3.

32. Lu R, Zhao X, Li J, et al. Genomic characterization and epidemiology of 2019 novel coronavirus: implications for virus origins and receptor binding. Lancet 2020;395:565-74.

33. Paraskevis D, Kostaki EG, Magiorkinis G, et al. Full-genome evolutionary analysis of the novel corona virus (2019-nCoV) rejects the hypothesis of emergence as a result of a recent recombination event. Infect Genet Evol 2020;79:104212.

34. $\mathrm{Hu} \mathrm{Z}$, Song $\mathrm{C}, \mathrm{Xu} \mathrm{C}$, et al. Clinical characteristics of 24 asymptomatic infections with COVID-19 screened among close contacts in Nanjing, China. Sci China Life Sci 2020. doi: 10.1007/s11427-020-1661-4

35. Lal A, Akhtar J, Jindal V et al. Rare cause of respiratory failure: A twist in the tale. Ann Am Thorac Soc 2018;15:880-3.

36. Lal A, Akhtar J, Isaac S, et al. Unusual cause of chest pain, Bornholm disease, a forgotten entity; case report and review of literature. Respir Med Case Rep 2018;25:270-3.

37. Chen L, Xiong J, Bao L, Shi Y. Convalescent plasma as a potential therapy for COVID-19. Lancet Infect Dis 2020. pii: S1473-3099(20)30141-9.

38. Ai T, Yang Z, Hou H, et al. Correlation of chest CT and RTPCR testing in Coronavirus Disease 2019 (COVID-19) in China: a report of 1014 cases. Radiology 2020;200642. doi: 10.1148/radiol.2020200642.

39. Yang Y, Yang M, Shen C, et al. Laboratory diagnosis and monitoring the viral shedding of 2019-nCoV infections. MedRxiv preprint 2020. doi: 10.1101/2020.02.11.20021493

40. Lal A, Abraham GM. Utility of CD4 cell count and viral load assay in hospitalized patients with known HIV infection: High value care exercise. Infect Disord Drug Targets 2019. [Epub ahead of print]. doi: 10.2174/1871526519666191011162018.

41. Mahdavi A, Khalili N, Davarpanah AH, et al. Radiologic management of COVID-19: Preliminary experience of the Iranian Society of Radiology COVID-19 Consultant Group (ISRCC). Iran J Radiol (In Press): e102324. doi: 10.5812/iranjradiol.102324.

42. Sahu KK, Mishra AK, Lal A. An update on CT chest findings in coronavirus disease-19 (COVID-19). Heart Lung 2020. doi: 10.1016/j.hrtlng.2020.03.007.

43. Zhou F, Yu T, Du R, et al. Clinical course and risk factors for mortality of adult inpatients with COVID-19 in Wuhan, China: a retrospective cohort study. Lancet 2020;395:1054-62.

44. Fan BE, Chong VCL, Chan SSW, et al. Hematologic parameters in patients with COVID-19 infection. Am J Hematol 2020. doi: 10.1002/ajh.25774

45. Mishra AK, Sahu KK, James A. Disseminated herpes zoster following treatment with benralizumab. Clin Respir J 2019;13:189-91.

46. Borchgrevink CP, Cha J, Kim S. Hand washing practices in a college town environment. J Environ Health 2013;75:18-24.

47. Yeo C, Kaushal S, Yeo D. Enteric involvement of coronaviruses: is faecal-oral transmission of SARS-CoV-2 possible? Lancet Gastroenterol Hepatol 2020. pii: S2468-1253(20)30048-0.

48. Xu J, Shi PY, Li H, Zhou J. Broad spectrum antiviral agent niclosamide and its therapeutic potential. ACS Infect Dis 2020. doi: 10.1021/acsinfecdis.0c00052

49. Yao TT, Qian JD, Zhu WY, et al. A systematic review of lopinavir therapy for SARS coronavirus and MERS coronavirus - A possible reference for coronavirus disease-19 treatment option. J Med Virol 2020. doi: 10.1002/jmv.25729

50. Andersen PI, Ianevski A, Lysvand H, et al. Discovery and development of safe-in-man broad-spectrum antiviral agents. Int J Infect Dis 2020. pii: S1201-9712(20)30076-X.

51. Wang M, Cao R, Zhang L, et al. Remdesivir and chloroquine effectively inhibit the recently emerged novel coronavirus (2019-nCoV) in vitro. Cell Res 2020;30:269-71.

52. Zumla A, Chan JF, Azhar EI, Hui DS, Yuen KY. Coronaviruses - drug discovery and therapeutic options. Nat Rev Drug Discov 2016;15:327-47.

53. Holshue ML, DeBolt C, Lindquist S, et al. First case of 2019 novel coronavirus in the United States. N Engl J Med 2020;382:929-36.

54. Sheahan TP, Sims AC, Leist SR, et al. Comparative therapeutic efficacy of remdesivir and combination lopinavir, ritonavir, and interferon beta against MERS-CoV. Nat Commun 2020;11:222.

55. Young BE, Ong SWX, Kalimuddin S, et al. Epidemiologic features and clinical course of patients infected with SARS-CoV2 in Singapore. JAMA 2020;e203204. doi: 10.1001/jama. 2020.3204

56. China CCDC. The Epidemiological characteristics of an outbreak of 2019 novel coronavirus diseases (COVID-19). China CCDC, February 17, 2020.

57. National Health Committee of the People's Republic of China, National Administration of Traditional Chinese Medicine. Diagnostic and therapeutic guidance for 2019 novel coronavirus disease (version 5). Available from: http://www. nhc.gov.cn/yzygj/s7653p/202002/d4b895337e19445f8d728fc af1e3e13a/files/ab6bec7f93e64e7f998d802991203cd6.pdf

58. Wang J, Guo Y, Li GL. Current status of standardization of 
Traditional Chinese Medicine in China. Evid Based Complement Alternat Med 2016;2016:9123103.

59. Sahu KK, Dhibar DP, Varma S, Malhotra P. CML with pregnancy: real challenges in developing nations. Leuk Lymphoma 2017;58:1518-9.

60. Liu Y, Chen H, Tang K, Guo Y. Clinical manifestations and outcome of SARS-CoV-2 infection during pregnancy. J Infect 2020. pii: S0163-4453(20)30109-2.

61. Favre G, Pomar L, Musso D, Baud D. 2019-nCoV epidemic: what about pregnancies? Lancet 2020;395: 40.

62. Sahu KK, Lal A, Mishra AK. COVID-2019 and pregnancy: A plea for transparent reporting of all cases. Acta Obstet Gynecol Scand 2020 Mar (Ahead of Pub).

63. Maternal and Fetal Experts Committee, Chinese Physician Society of Obstetrics and Gynecology, Chinese Medical Doctor Association, Obstetric Subgroup, et al. Proposed management of 2019-novel coronavirus infection during pregnancy and puerperium. Chin J Perinat Med 2020;23:73-9.

64. Sahu KK, Varma SC. Herpes zoster complicating bortezomib therapy. Indian J Med Res 2015;141:247-8.

65. Sahu KK, Prakash G, Khadwal A, et al. A rare case of hemor- rhagic cystitis in allogeneic hematopoietisac stem cell transplant patient. Indian J Hematol Blood Transfus 2016;32:196-200.

66. Sahu KK, Jindal V, Siddiqui AD, Cerny J. Facing COVID-19 in the hematopoietic cell transplant setting: A new challenge for transplantation physicians. Blood Cell Mod Dis 2020. doi: 10.1016/j.bcmd.2020.102439.

67. Mishra AK, Sahu KK, Lal A, Sargent J. Patterns of heart injury in COVID-19 and relation to outcome. J Med Virol 2020. doi: 10.1002/jmv.25847.

68. Lal A, Mishra AK, Sahu KK. CT chest findings in coronavirus disease-19 (COVID-19). J Formos Med Assoc 2020. pii: S09296646(20)30102-9.

69. Lal A, Mishra AK, Sahu KK. Is EVALI the new scapegoat for every abnormal chest imaging? Heart Lung 2020. pii: S01479563(20)30019-4. doi: 10.1016/j.hrtlng.2020.02.005.

70. Lal A, Mishra AK, Sahu KK. Vitamin E acetate and E-Cigarette or Vaping Product-Associated Lung Injury (EVALI): An update. Am J Med 2019. doi: 10.1016/j.amjmed.2019.11.005

71. Mishra AK, Sahu KK, Lal A. Reporting of all cardiac medications and their outcome in COVID - 19. J Med Virol 2020. doi: 10.1002/jmv.25843. 